\title{
Optimization of Hybrid Hub-and-Spoke Network Operation for Less-Than-Truckload Freight Transportation considering Incremental Quantity Discount
}

\author{
Weiya Chen, Kaixian He, and Xiaoping Fang \\ School of Traffic and Transportation Engineering, Central South University, Changsha 410075, China \\ Correspondence should be addressed to Xiaoping Fang; fangxp@csu.edu.cn
}

Received 10 July 2014; Accepted 16 September 2014; Published 29 September 2014

Academic Editor: Huimin Niu

Copyright (c) 2014 Weiya Chen et al. This is an open access article distributed under the Creative Commons Attribution License, which permits unrestricted use, distribution, and reproduction in any medium, provided the original work is properly cited.

\begin{abstract}
This paper presents a mixed integer linear programming model (MILP) for optimizing the hybrid hub-and-spoke network operation for a less-than-truckload transportation service. The model aims to minimize the total operation costs (transportation cost and transfer cost), given the determined demand matrix, truck load capacity, and uncapacitated road transportation. The model also incorporates an incremental quantity discount function to solve the reversal of the total cost and the total demand. The model is applied to a real case of a Chinese transportation company engaged in nationwide freight transportation. The numerical example shows that, with uncapacitated road transportation, the total costs and the total vehicle trips of the hybrid hub-and-spoke network operation are, respectively, $8.0 \%$ and $15.3 \%$ less than those of the pure hub-and-spoke network operation, and the assumed capacity constraints in an extension model result in more target costs on the hybrid hub-and-spoke network. The two models can be used to support the decision making in network operations by transportation and logistics companies.
\end{abstract}

\section{Introduction}

Transportation companies usually have their own physical operation network with facilities located in different levels of nodes. Generally there are three kinds of physical operation network: direct transportation network, pure hub-andspoke network, and hybrid hub-and-spoke network. It is a great challenge for transportation companies to make daily decisions about optimal network operations on an existing physical network, especially on a hub-and-spoke one.

A direct transportation network is a fully connected network which has the advantage of short transport distance and fast speed [1]. However, in terms of LTL (less-thantruckload) transportation, it is faced with the risk of losing economies of scale since not every origin-destination (OD) flow can stably satisfy the effective truck load capacity. The fact is that some small and unstable OD flows result in high operation cost, and even a high risk of partial loss. Overcoming the above disadvantages, the hub-and-spoke network makes use of economies of scale on trunk road transport by collecting goods to pivots $[2,3]$. Although it inevitably sometimes produces bypass transportation and transfer processing costs, in order to cover a wider marketing, some transportation and logistics companies prefer to adopt the hub-and-spoke network [4].

To help with daily operational decision making, a lot of research has been done to optimize the hub-and-spoke network operation. In these research works, mathematical models and algorithms are used to optimize pure hub-andspoke network operations in three aspects: hub location optimization, routing optimization, and both [5-12]. In a pure hub-and-network, all OD flows in between nonhub nodes need a transfer operation at least once. Demand flows between different OD pairs usually increase unevenly; however, some of the OD flows may achieve the effective scale of direct transport or need a transfer operation only once. This unnecessarily produces more transportation and transfer costs and delays the arrival time. Thus, a hybrid hub-and-spoke network is used to overcome these problems. 
To optimize the hybrid hub-and-spoke network operation, different models, formulations, and algorithms have been addressed in numerous papers in the literature. Liu and colleagues investigated a mixed truck delivery system that allows both hub-and-spoke and direct shipment and used a heuristic algorithm to determine the delivery modes and vehicle routings [13]. Zapfel and Wasner presented a mathematical model to optimize the mixed hub-and-spoke network by minimizing the total costs of transportation cost and variable sorting cost based on a physical network [14]. Barcos and colleagues designed an LTL cargo operation network by minimizing the total transportation costs and transfer processing costs, guaranteeing a certain service level and allowing for vehicle routes with multiple unloading stations [15]. The latter two papers show that hybrid huband-spoke networks are more efficient and economical than pure hub-and-spoke networks as they do not consider the reconstruction and renovation cost of the physical network. More specifically, Adler investigated hub-spoke network operations in a competitive environment [16]. Elhedhli and $\mathrm{Hu}$ designed a hybrid hub-and-spoke network which took congestion into account [17]. Matsubayashi and colleagues studied the cost allocation problem in a hybrid hub-andspoke network system [18]. These works showed that the hybrid hub-and-spoke network has advantages in terms of cost savings and equipment efficiency over the pure hub-andspoke network.

Another noteworthy fact is that, in order to attract more order volumes, different price discount policies are used by transportation companies [19]. The most commonly used discount policy is unified discount. The larger the order volume is, the higher the price discount is. It allows customers to adjust their order volumes to reach a higher price discount point. One shortcoming of this pricing policy is that it leads to a reversal of the total cost and the total flow near some discount points, which often causes confusion or even conflict among transportation companies and their customers.

This paper aims to design and optimize the operation of a hybrid hub-and-spoke network by employing an existing physical hub-and-spoke network. Direct transport between different nodes is allowed if the OD flow reaches an effective scale. Otherwise the OD flows in between nontransfer nodes need to be transferred at hubs at least once. Vehicle routes are optimized and an incremental quantity discount function is embedded to make sure that the total cost is increasing strictly by total demand.

The remainder of this paper is organized as follows. Section 2 presents a mixed integer linear programming model (MILP) to optimize the LTL transportation operation of a hybrid hub-and-spoke network with uncapacitated road segments taking into account the incremental quantity discount. Section 3 shows an application of this model to the real case of a Chinese logistic company engaged in nationwide freight transportation. Section 4 formulates an extension model considering capacitated road segments, namely, links, and presents numerical results. Conclusions are drawn in Section 5 .

\section{Model Formulation}

The model focuses on the optimization of an existing physical network in which less-than-truckload freight transportation services are provided by a transportation company. This physical network consists of a set of transfer nodes and a set of nontransfer nodes, namely, hubs and nonhub nodes in this paper. Let a complete graph $G(N, A)$ be the physical network, where $N$ is the set of all nodes (specifically, $S$ is the set of hubs, which is a subset of $N$ ) and $A$ is the set of links. Given the OD matrix and a discount policy, the model aims to generate an optimal operational network based on the graph $G(N, A)$. Before the model is constructed, some assumptions are made as follows.

(1) The OD flows are determined, splittable and allowed to be directly transported, and transferred once or twice.

(2) The load capacity of each truck is fixed. The service frequency is determined by the goods flows and subject to a promised service level.

(3) The total operation costs consist of transportation cost and transfer cost. Transportation cost is related to the flow, which is characterized by an incremental quantity discount function $f(\cdot)$. Transfer cost at hubs usually consists of two parts: fixed transfer cost and variable transfer cost. In this paper, since we focus on the optimization problem of an existing physical network, the fixed transfer cost is ignored and the transfer cost is equal to the variable transfer cost, denoted by function $g(\cdot)$.

(4) The hubs and road segments are uncapacitated.

Other notations are as follows:

$h_{i j}$ : total flow (by weight) from node $i$ to node $j$;

$c_{k m}$ : unit transportation cost (per kilogram per kilometer) of link $k-m$;

$F_{k m}^{r}$ : flow of link $k-m$ that adopts discount of interval $r$, namely, link flow. The domain of the whole discount function is divided into $R$ intervals, $r=1,2, \ldots, R$;

$d_{k m}$ : distance from node $k$ to node $m$;

$F_{k}$ : flow transferred at node $k$, namely, transfer flow;

$\lambda_{k}$ : unit transfer cost at node $k$;

$y_{k m}^{r}$ : switch function, valued as one or zero, respectively, means link $k-m$ uses discount of interval $r$ or not;

$X_{i j}^{k m}$ : proportion of goods flows which take the route $i-k-m-j$ to all goods flows from node $i$ to $j$. Thus, $i-i-i-j, i-i-j-j$, and $i-j-j-j$ are all equal to the direct route $i-j . i-i-k-j, i-k-k-j$, and $i-k-j-j$ are all equal to the route $i-k-j$.

In this study, the optimization target is to minimize the total operation costs $C$, which can be denoted as the following objective function:

$$
\min C=\sum_{k \in N} \sum_{m \in N} \sum_{r} f\left(F_{k m}^{r}\right)+\sum_{k \in S} g\left(F_{k}\right) .
$$


In the right side of (1), the first part is the total transportation cost, which is a function of the link flow $F_{k m}^{r}$; the second part is the total transfer cost at hubs, which is a function of the transfer flow $F_{k}$. In order to solve the reversal of the total cost and the total flow, an incremental quantity discount $\alpha$ is used in this paper, which is distinct from thresholdbased discounting [19]. The incremental quantity discount $\alpha$ is decided by

$$
\alpha= \begin{cases}\alpha_{1}, & l_{1} \leq F_{k m}^{1} \leq u_{1} \\ \alpha_{2}, & l_{2} \leq F_{k m}^{2} \leq u_{2} \\ \vdots & \\ \alpha_{R}, & l_{R} \leq F_{k m}^{R} \leq u_{R},\end{cases}
$$

where $l_{r}$ and $u_{r}$ are the lower and upper bound of interval $r$, respectively, and $l_{1}=0, u_{1}=l_{2}, \ldots, u_{R-1}=l_{R} ; \alpha_{R}$ is the discount rate of interval $\left[l_{R}, u_{R}\right]$. Commonly, a decreasing discount rate is used to attract more freight volumes; thus let $\alpha_{R}<\alpha_{R-1}<\cdots<\alpha_{1}$. Then we can obtain the average unit discount $\bar{\alpha}$ in

$$
\bar{\alpha}= \begin{cases}\frac{\alpha_{1},}{\alpha_{1}\left(u_{1}-l_{1}\right)+\alpha_{2}\left(F_{k m}^{2}-l_{2}\right)} & l_{1} \leq F_{k m}^{1} \leq u_{1} \\ \vdots & l_{2} \leq F_{k m}^{2} \leq u_{2} \\ \left(\alpha_{1}\left(u_{1}-l_{1}\right)+\alpha_{2}\left(u_{2}-l_{2}\right)\right. & \\ \left.+\cdots+\alpha_{R}\left(F_{k m}^{R}-l_{R}\right)\right) \times\left(F_{k m}^{R}\right)^{-1}, & l_{R} \leq F_{k m}^{R} \leq u_{R} .\end{cases}
$$

In (3), $\bar{\alpha}$ is the weighted average unit discount rate of one customer's total freight volume. When $\alpha_{R}<\alpha_{R-1}<\cdots<\alpha_{1}$ and the discount interval is set properly, the larger the total volume is, the smaller the weighted average unit discount rate will be. Under this discounting policy, transportation or logistics companies can use quantity discounting to attract more order volume; while customers could adjust their order volume to obtain a self-satisfied average unit discount point to save cost, but their total cost will still rise along with the rising total volume.

Note that transportation cost also has a relation with the transportation distance. By incorporating the average unit discount rate $\bar{\alpha}$, we can formulize the transportation cost as

$$
f\left(F_{k m}^{r}\right)=\bar{\alpha} c_{k m} F_{k m}^{r} d_{k m} .
$$

For simplicity, the transfer cost is assumed to be proportional to the transfer flow at hubs. The transfer cost at node $k$ is formulized as

$$
g\left(F_{k}\right)=\lambda_{k} F_{k} .
$$

Thus, we can rewrite objective function (1) into

\begin{tabular}{|c|c|}
\hline Hubs & Nonhub nodes \\
\hline Beijing (BJ) & $\begin{array}{l}\text { Tianjin (TJ), Shijiazhuang (SJZ), Jinan } \\
\text { (JN), Harbin (HEB), Changchun (CC), } \\
\text { and Shenyang (SY) }\end{array}$ \\
\hline Zhengzhou (ZZ) & Taiyuan (TY) and Xi'an (XA) \\
\hline Wuhan (WH) & Changsha (CS) \\
\hline Guangzhou (GZ) & Chengdu (CD) \\
\hline Shanghai (SH) & $\begin{array}{l}\text { Nanjing (NJ), Hangzhou (HZ), and } \\
\text { Fuzhou (FZ) }\end{array}$ \\
\hline
\end{tabular}

$$
\begin{aligned}
\min C= & \sum_{k \in N} \sum_{m \in N} \sum_{r} \bar{\alpha} c_{k m} F_{k m}^{r} d_{k m} \\
& +\sum_{k} \lambda_{k}\left\{\sum_{i, j} h_{i j}\left[\sum_{m}\left(X_{i j}^{k m}+X_{i j}^{m k}\right)-X_{i j}^{k k}\right]\right\}
\end{aligned}
$$

TABLE 1: Hubs and nonhub nodes.

subject to

$$
\begin{gathered}
\sum_{r} F_{k m}^{r}=\sum_{i, j} h_{i j} X_{i j}^{k m}+\sum_{i, j} h_{k i} X_{k i}^{m j}+\sum_{i \neq k \cup j \neq m} h_{i m} X_{i m}^{j k}, \quad \forall k, m \\
F_{k m}^{r}-y_{k m}^{r} l_{r} \geq 0, \quad \forall k, m, r, \\
F_{k m}^{r}-y_{k m}^{r} u_{r} \leq 0, \quad \forall k, m, r \\
\sum_{r} y_{k m}^{r}=1, \quad \forall k, m, \\
\sum_{k} \sum_{m} X_{i j}^{k m}=1, \quad \forall i, j, \\
X_{i j}^{k m} \geq 0, \quad \forall i, j, k, m, \\
X_{i j}^{k m}=0, \quad k \notin \mathbb{S} \cup m \notin \mathbb{S} \\
y_{k m}^{r}=\{0,1\} \quad \forall k, m .
\end{gathered}
$$

Constraint (7) ensures that, if $m=k$, the route $i-k-k-j$ will be calculated just once. Constraints (8) and (9) ensure that $F_{k m}^{r}$ will be located in the interval $\left[l_{r}, u_{r}\right]$. Constraint (11) ensures the equilibrium of all flows. Constraint (12) ensures that the goods flows are nonnegative. Constraint (13) ensures that the transferred flows can just be transferred at hubs. Constraints (10) and (14) ensure the consistency of the discount rate.

\section{Model Application and Solution}

3.1. Sample Data. The data come from a transportation company in China which is engaged in LTL freight transportation in 18 cities. Of those cities, five have transfer hubs and the rest are nonhub nodes. All nodes of cities in the physical network are shown in Table 1 . We extract sample data covering all the business in the 18 cities for 2011, comprising 128018 records. The summed OD flows by weight are shown in Table 3 . We take an average capacity of 28 tons for each truck in the following calculation. 
TABLE 2: Comparison of total cost and computing time of different hub sets.

\begin{tabular}{lcc}
\hline Hubs & Cost/Yuan & Time/s \\
\hline 1 & $1.63304 e 7$ & 17 \\
2 & $1.19642 e 7$ & 93 \\
3 & $1.08919 e 7$ & 404 \\
4 & $9.66232 e 6$ & 7543 \\
5 & $7.99414 e 6$ & 36453 \\
\hline
\end{tabular}

The incremental quantity discount policy is given as follows:

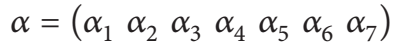

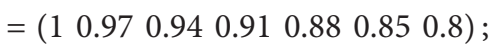

$$
\begin{aligned}
& l_{r}=\left(\begin{array}{lllllll}
l_{1} & l_{2} & l_{3} & l_{4} & l_{5} & l_{6} & l_{7}
\end{array}\right) \\
& =\left(\begin{array}{lllllll}
0 & 20 & 40 & 60 & 80 & 100 & 120
\end{array}\right) \text {; } \\
& u_{r}=\left(\begin{array}{lllllll}
u_{1} & u_{2} & u_{3} & u_{4} & u_{5} & u_{6} & u_{7}
\end{array}\right) \\
& =\left(\begin{array}{lllllll}
20 & 40 & 60 & 80 & 100 & 120 & 999999
\end{array}\right) \text {, }
\end{aligned}
$$

where $u_{7}=999999$ means a very large value for running the calculations correctly. This incremental quantity discount policy is characterized by an incremental quantity of 20 tons with a decreasing discount rate of 0.03 with an exception of 0.05 for $\alpha_{7}$, which is adjusted according to the sample company's marketing pricing strategy.

3.2. Calculations. The model of formulae (6) to (14) is a mixed integer linear programming model. An exponential algorithm in Lingo 11.0 can be used to solve this mixed integer programming problem. The computational results are shown in Table 4.

As regards the pure hub-and-spoke network and the hybrid hub-and-spoke network, respectively, the total costs are 8686950 and 7994138 Yuan, and the total trips are 145974 and 123601 . Reductions of $8.0 \%$ and $15.3 \%$ in costs and trips apply to the hybrid hub-and-spoke network compared with the pure hub-and-spoke network. The optimized operation network is shown in Figure 1.

Lingo 11.0 is executed by a desktop with the CPU environment of AMD Athlon $64 * 2$ Dual-Core Processor TK53. The computing time increases greatly as the number of hubs increases. The results are listed in Table 2.

\section{Model Extension: Capacitated Hybrid Hub-and-Spoke Network Operation for LTL Freight Transportation}

When goods flow is increasing, the capacity constraint should be taken into consideration. We take the road capacity, hub capacity, and truck capacity as one unified capacity constraint, namely, link capacity on a graph, because these kinds of capacity in a physical network finally restrain the flows in the link. Consequently, an extension model based on the model in Section 2 is constructed. Mathematically, we obtain the following extension model:

$$
\begin{aligned}
\min C= & \sum_{k \in N} \sum_{m \in N} \sum_{r} \bar{\alpha} c_{k m} F_{k m}^{r} d_{k m} \\
& +\sum_{k} \lambda_{k}\left\{\sum_{i, j} h_{i j}\left[\sum_{m}\left(X_{i j}^{k m}+X_{i j}^{m k}\right)-X_{i j}^{k k}\right]\right\}
\end{aligned}
$$

subject to

$$
\begin{gathered}
\sum_{r} F_{k m}^{r}=\sum_{i, j} h_{i j} X_{i j}^{k m}+\sum_{i, j} h_{k i} X_{k i}^{m j}+\sum_{i \neq k \cup j \neq m} h_{i m} X_{i m}^{j k}, \quad \forall k, m \\
\sum_{r} F_{k m}^{r} \leq C_{k m}, \quad \forall k, m, \\
F_{k m}^{r}-y_{k m}^{r} l_{r} \geq 0, \quad \forall k, m, r \\
F_{k m}^{r}-y_{k m}^{r} u_{r} \leq 0, \quad \forall k, m, r \\
\sum_{r} y_{k m}^{r}=1, \quad \forall k, m, \\
\sum_{k} \sum_{m} X_{i j}^{k m}=1, \quad \forall i, j, \\
X_{i j}^{k m} \geq 0, \quad \forall i, j, k, m, \\
X_{i j}^{k m}=0, \quad k \notin \mathbb{S} \cup m \notin \mathbb{S} \\
y_{k m}^{r}=\{0,1\} \quad \forall k, m .
\end{gathered}
$$

In (18), $C_{k m}$ is the upper limit of the flow in link $k-m$. Since the road segments are not subject to any capacity constraint in the sample enterprise, all the segments are assumed to have an upper limit of 3.1 million tons per month. The optimized network is presented in Figure 2.

Compared with the uncapacitated model, when the flow exceeds the link capacity and these kinds of links are few, the operational network does not change very much. The goods flows sent by each link computed by the two models are listed in Tables 4 and 5. However, the total costs have changed. The total costs are 8065921 Yuan, $0.90 \%$ higher than those of the uncapacitated network. The main reason is that the goods flow exceeding the road capacity is sent by other links because of insufficient capacity. In the example, each link has an upper capacity of 3.1 million tons per month, and only four links are fully used in the optimized network (see the red links in Figure 2).

\section{Conclusions}

Operation optimization is critical for reducing operation costs for transportation companies engaged in nationwide LTL freight transportation in an existing physical hub-andspoke network. In this paper, a MILP model for LTL transportation operation optimization of a hybrid hub-and-spoke network is proposed which takes into account an incremental 


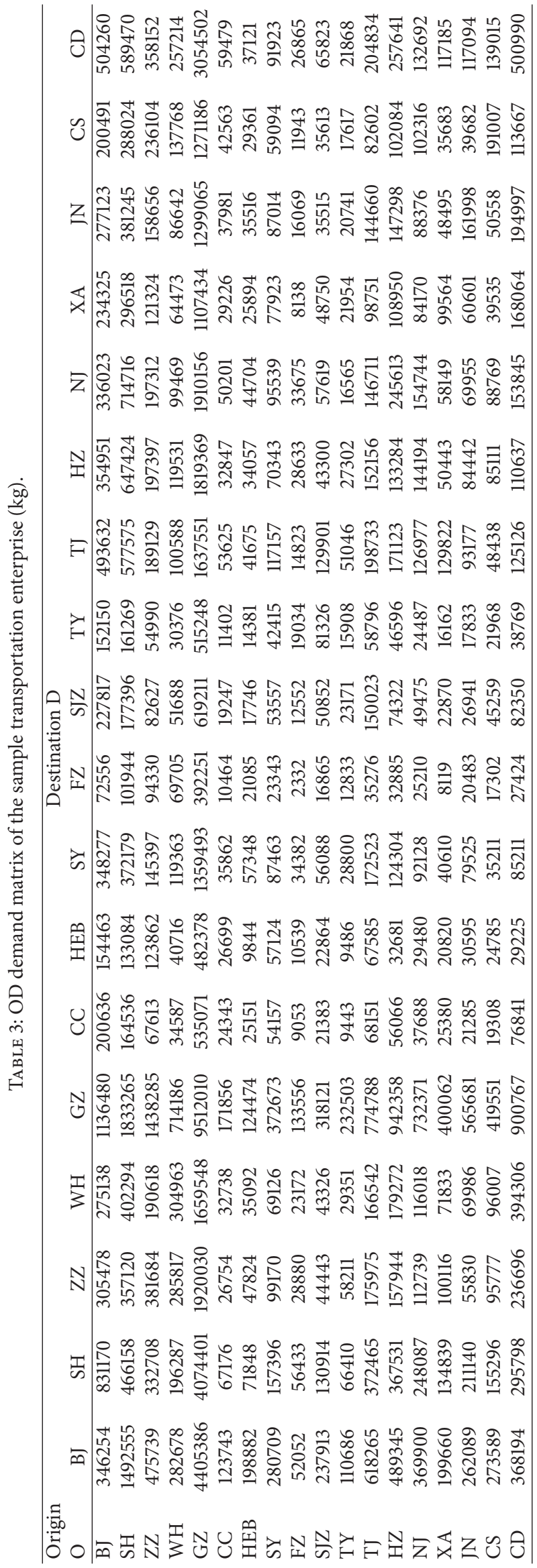




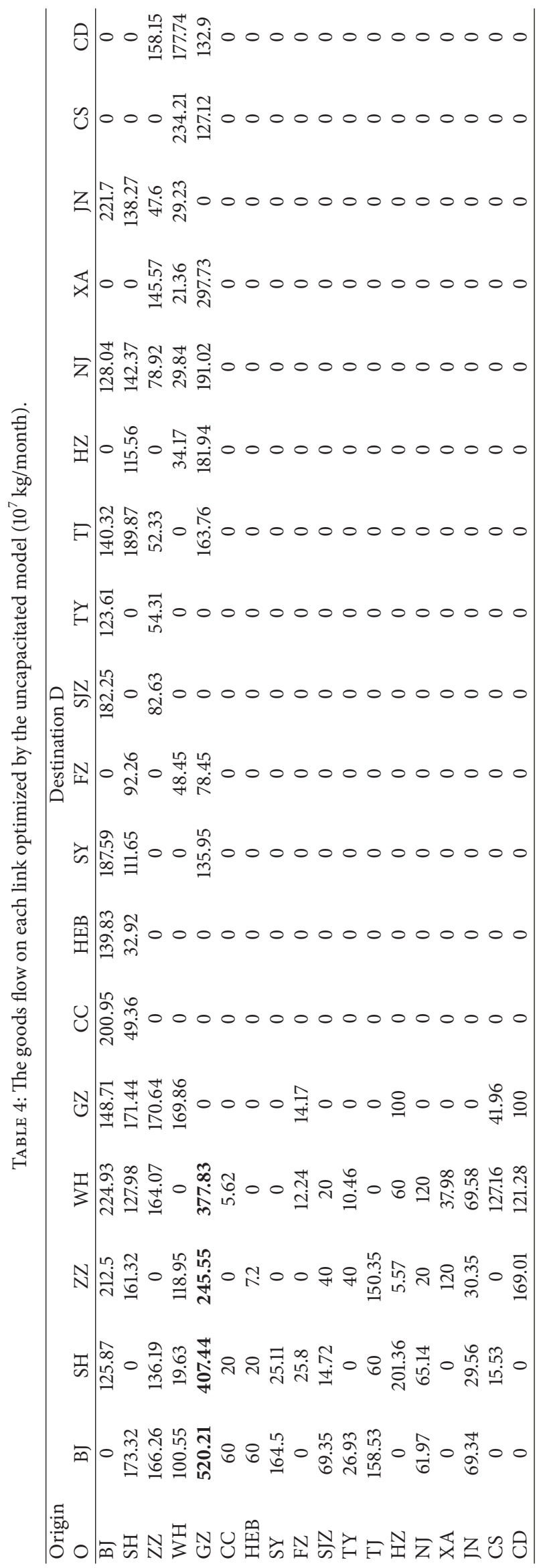




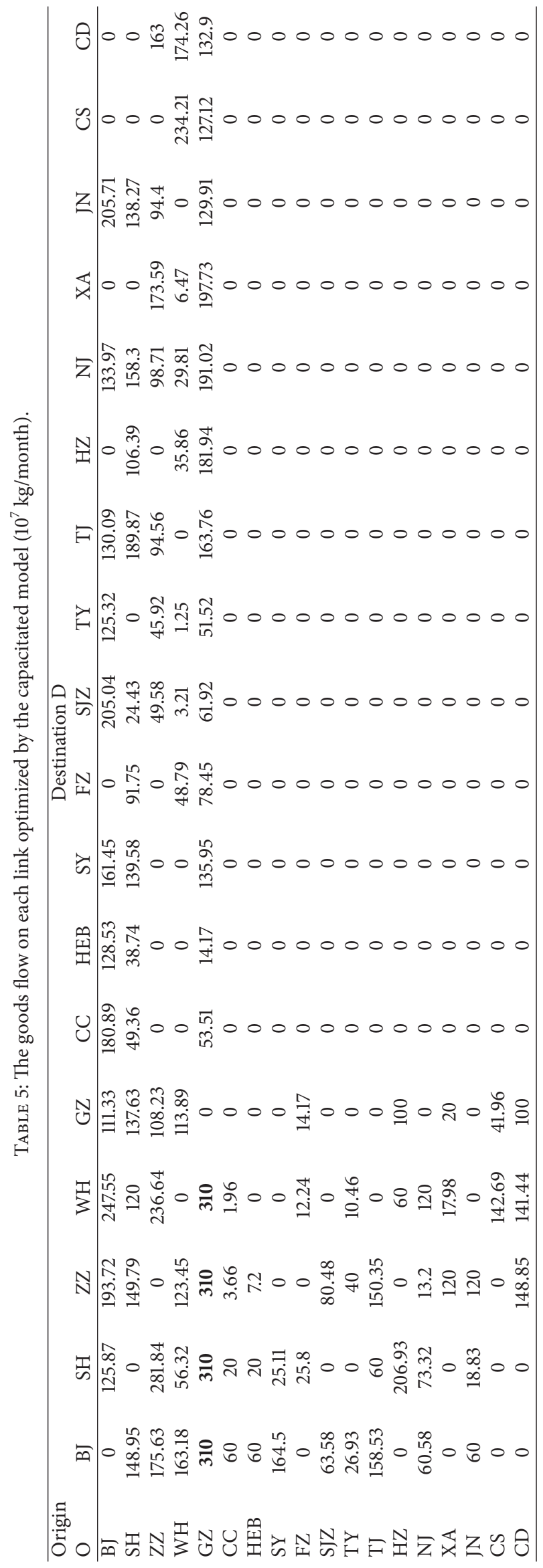




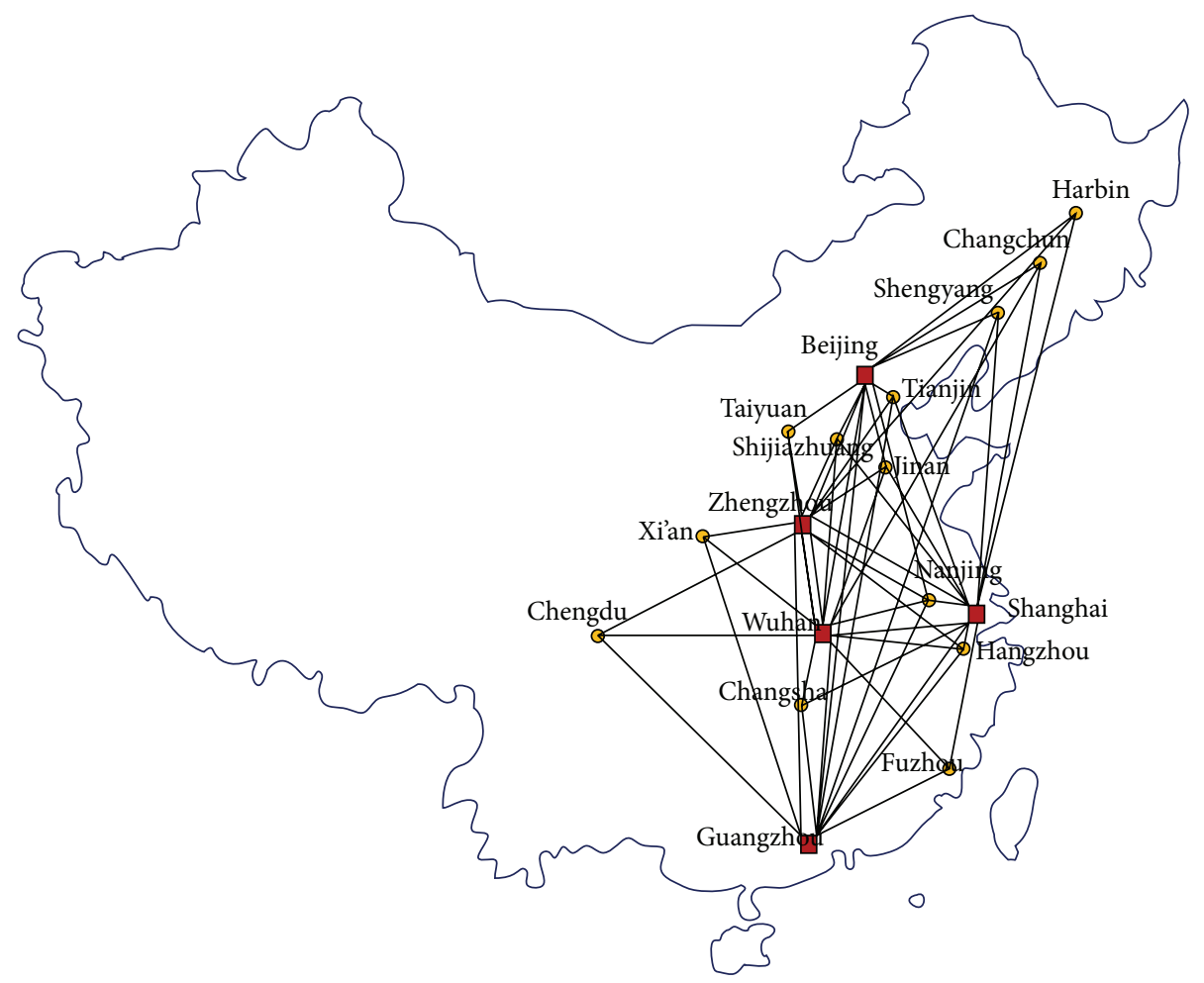

Hubs

O Nonhub nodes

Figure 1: Optimized uncapacitated hybrid hub-and-spoke network for LTL freight transportation.

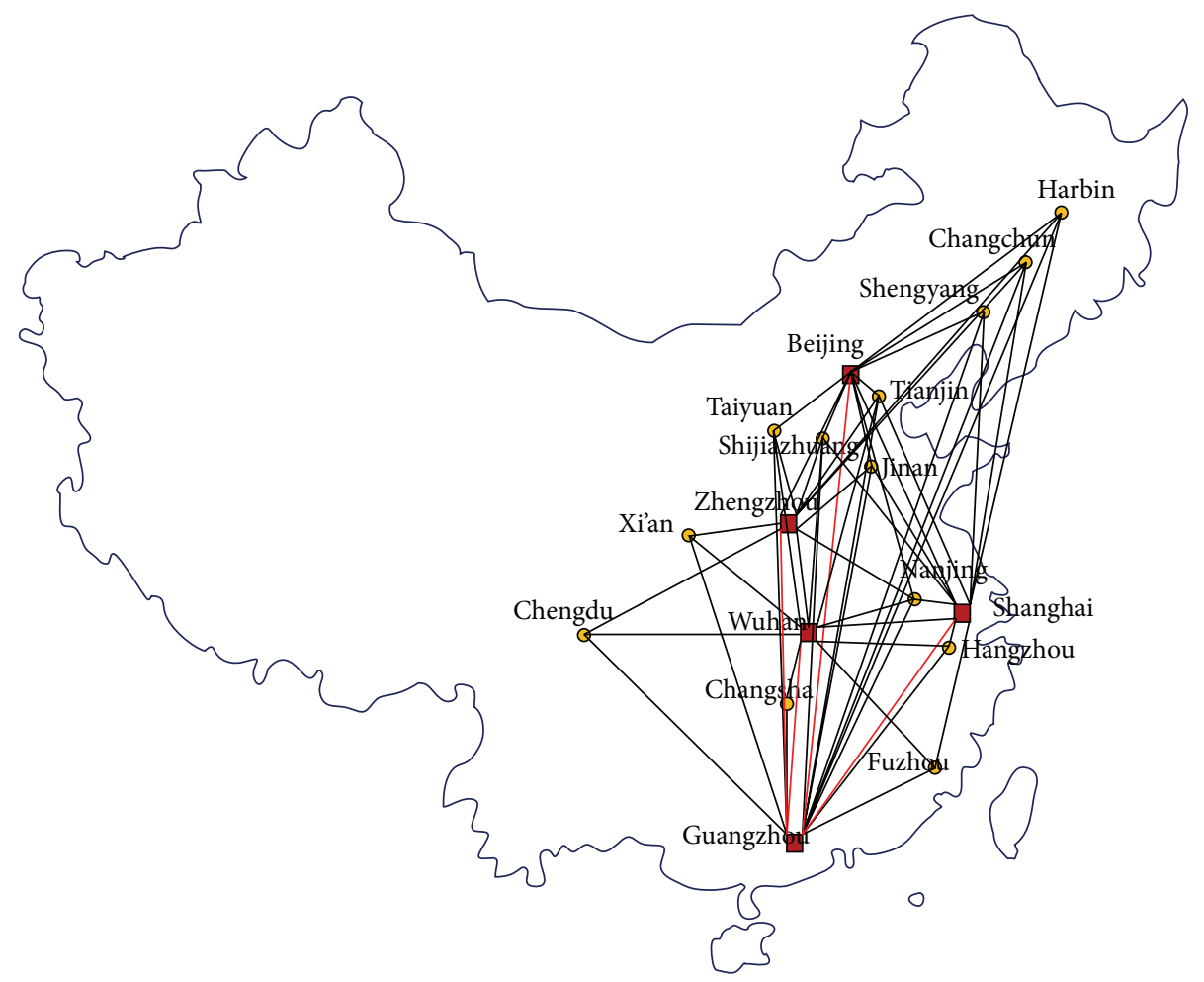

Hubs

O Nonhub nodes

FIGURE 2: Optimized capacitated hybrid hub-and-spoke network for LTL freight transportation. 
quantity discount function in order to solve the reversal of the total costs and the total flow. In fact, for a company engaged in nationwide transportation in an existing hub-and spoke network, when the OD flows are not big enough for direct transport, a hub-and-spoke system can help to obtain economies of scale. The results of the model application for the sample enterprise indicate that $56.21 \%$ of the goods flows are sent by direct transport, $32.15 \%$ of which are sent from one depot to another; $27.95 \%$ of the goods flows are sent and transferred once at a hub, and only $15.84 \%$ are transferred twice; the hybrid hub-and-spoke network operation saves $8.0 \%$ more on total costs and $15.3 \%$ more on total trips than the pure hub-and-spoke network does. Furthermore, the incremental quantity discount price policy can eliminate the reversal of the total cost and the total transport volume.

In daily management, trucks are seldom dispatched between different pairs of OD except in case of emergency or by request. Thus, we have to take capacity constraint into account. In the extension model in this paper, the assumed capacity constraint increases the total costs. The main reason is that the excess OD flow has to be sent via bypass transport, at higher cost. This extension model can be used to support business owners in making decisions on optimizing facilities.

The proposed models can be applied to logistic companies and cargo transportation companies with an existing physical hub-and-spoke network. The models adapt well to different numbers of hubs and the OD flow matrix. They can be used to support daily decision-making in the case of changing OD flow matrices.

\section{Appendix}

See Tables 3, 4, and 5 .

\section{Conflict of Interests}

The authors declare that there is no conflict of interests regarding the publication of this paper.

\section{Acknowledgments}

This research was supported by the National Natural Science Foundation of China (NSFC) under Grant no. 61203162 and the National Social Science Foundation of China under Grant no. 11CGL032. This research is also supported in part by the Research and Development Funds of Science and Technology of China Railway Corporation (2013X008) and Social Science Foundation of China Education Ministry (11YJC790143).

\section{References}

[1] C. C. Lin, Y. J. Lin, and D. Y. Lin, "The economic effects of center-to-center directs on hub-and-spoke networks for air express common carriers," Journal of Air Transport Management, vol. 9, no. 4, pp. 255-265, 2003.

[2] M. E. O'Kelly and D. L. Bryan, "Hub location with flow economies of scale," Transportation Research Part B: Methodological, vol. 32, no. 8, pp. 605-616, 1998.
[3] M. W. Horner and M. E. O'Kelly, "Embedding economies of scale concepts for hub network design," Journal of Transport Geography, vol. 9, no. 4, pp. 255-265, 2001.

[4] G. Nero and J. A. Black, "Hub-and-spoke networks in the inclusion of environmental costs on airport pricing," Transportation Research Part D: Transport and Environment, vol. 3, no. 5, pp. 275-296, 1998.

[5] J. F. Campbell, "Integer programming formulations of discrete hub location problems," European Journal of Operational Research, vol. 72, no. 2, pp. 387-405, 1994.

[6] J. Ebery, M. Krishnamoorthy, A. Ernst, and N. Boland, "Capacitated multiple allocation hub location problem: formulations and algorithms," European Journal of Operational Research, vol. 120, no. 3, pp. 614-631, 2000.

[7] A. Marín, "Formulating and solving splittable capacitated multiple allocation hub location problems," Computers and Operations Research, vol. 32, no. 12, pp. 3093-3109, 2005.

[8] H. Yaman, B. Y. Kara, and B. C.. Tansel, "The latest arrival hub location problem for cargo delivery systems with stopovers," Transportation Research Part B: Methodological, vol. 41, no. 8, pp. 906-919, 2007.

[9] A. T. Ernst, H. Hamacher, H. Jiang, M. Krishnamoorthy, and G. Woeginger, "Uncapacitated single and multiple allocation phub center problems," Computers and Operations Research, vol. 36, no. 7, pp. 2230-2241, 2009.

[10] R. Z. Farahani, M. Hekmatfar, A. B. Arabani, and E. Nikbakhsh, "Hub location problems: a review of models, classification, solution techniques, and applications," Computers and Industrial Engineering, vol. 64, no. 4, pp. 1096-1109, 2013.

[11] S. Melkote and M. S. Daskin, "An integrated model of facility location and transportation network design," Transportation Research Part A: Policy and Practice, vol. 35, no. 6, pp. 515-538, 2001.

[12] D. Sun and L. Elefteriadou, "Research and implementation of lane-changing model based on driver behavior," Transportation Research Record, no. 2161, pp. 1-10, 2010.

[13] J. Liu, C.-L. Li, and C.-Y. Chan, "Mixed truck delivery systems with both hub-and-spoke and direct shipment," Transportation Research Part E: Logistics and Transportation Review, vol. 39, no. 4, pp. 325-339, 2003.

[14] G. Zapfel and M. Wasner, "Planning and optimization of hub-and-spoke transportation networks of cooperative thirdparty logistics providers," International Journal of Production Economics, vol. 78, no. 2, pp. 207-220, 2002.

[15] L. Barcos, V. M. Rodriguez, M. J. Alvarez, and F. Robuste, "Routing design for less-than-truckload motor carriers using ant colony techniques," Workings Paper, Business Economics Series 14, Universidad Carlos III, 2004.

[16] N. Adler, "Hub-spoke network choice under competition with an application to Western Europe," Transportation Science, vol. 39, no. 1, pp. 58-72, 2005.

[17] S. Elhedhli and F. X. Hu, "Hub-and-spoke network design with congestion," Computers and Operations Research, vol. 32, no. 6, pp. 1615-1632, 2005.

[18] N. Matsubayashi, M. Umezawa, Y. Masuda, and H. Nishino, "A cost allocation problem arising in hub-spoke network systems," European Journal of Operational Research, vol. 160, no. 3, pp. 821-838, 2005.

[19] H. Podnar, J. Skorin-Kapov, and D. Skorin-Kapov, "Network cost minimization using threshold-based discounting," European Journal of Operational Research, vol. 137, no. 2, pp. 371-386, 2002. 


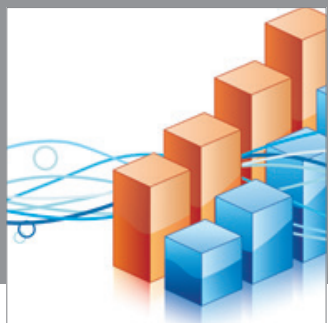

Advances in

Operations Research

mansans

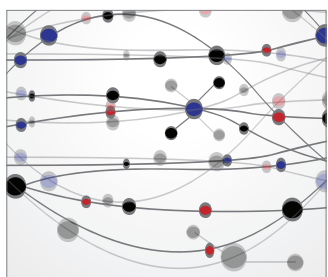

The Scientific World Journal
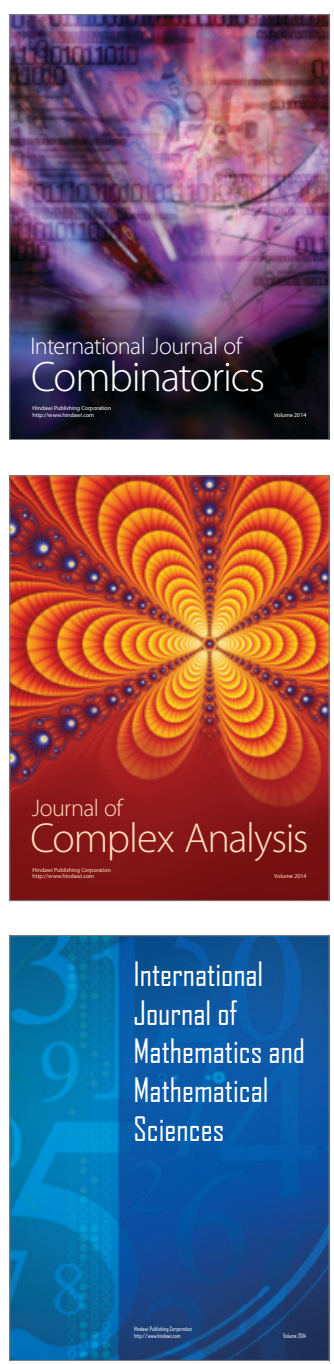
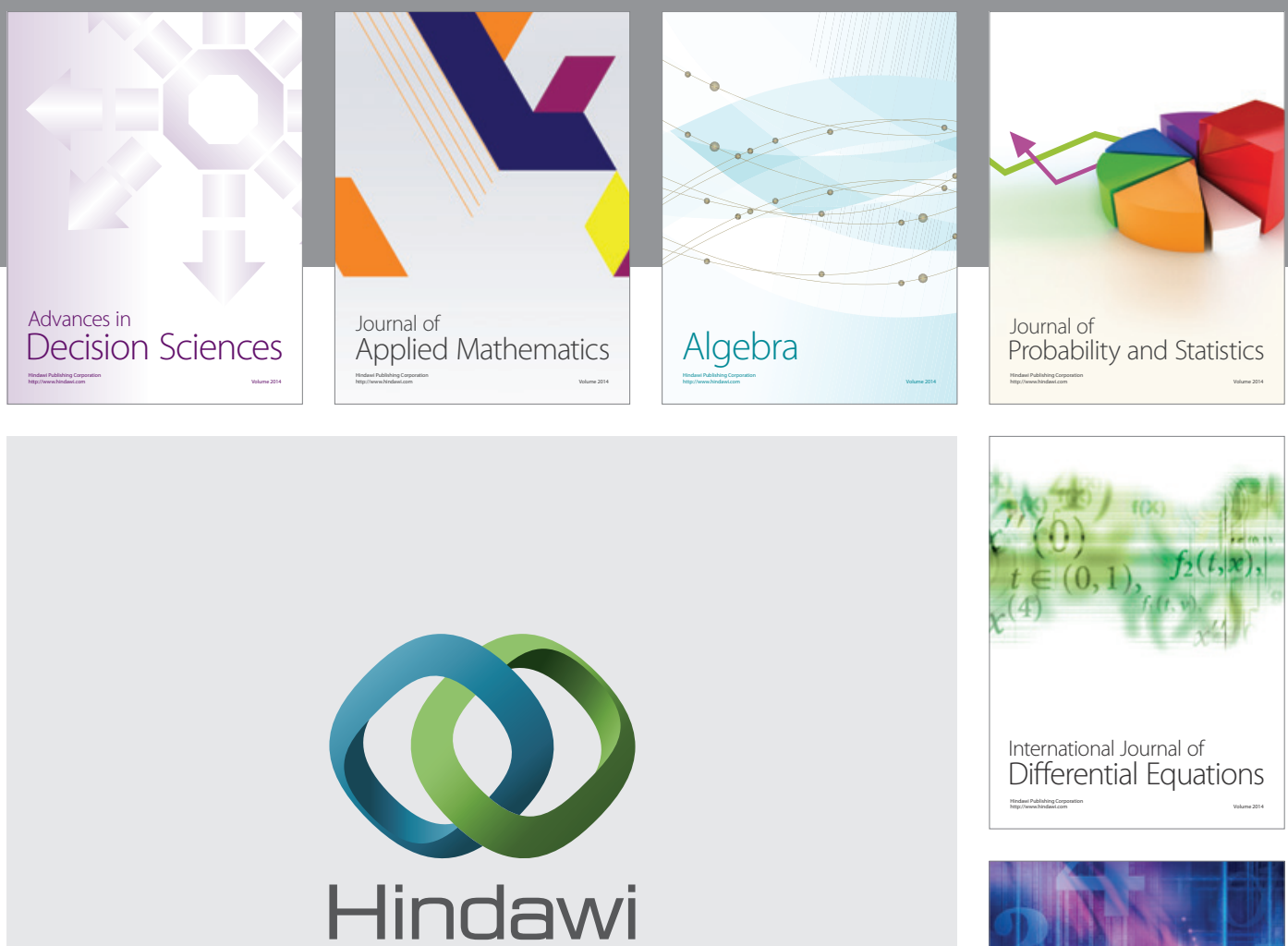

Submit your manuscripts at http://www.hindawi.com
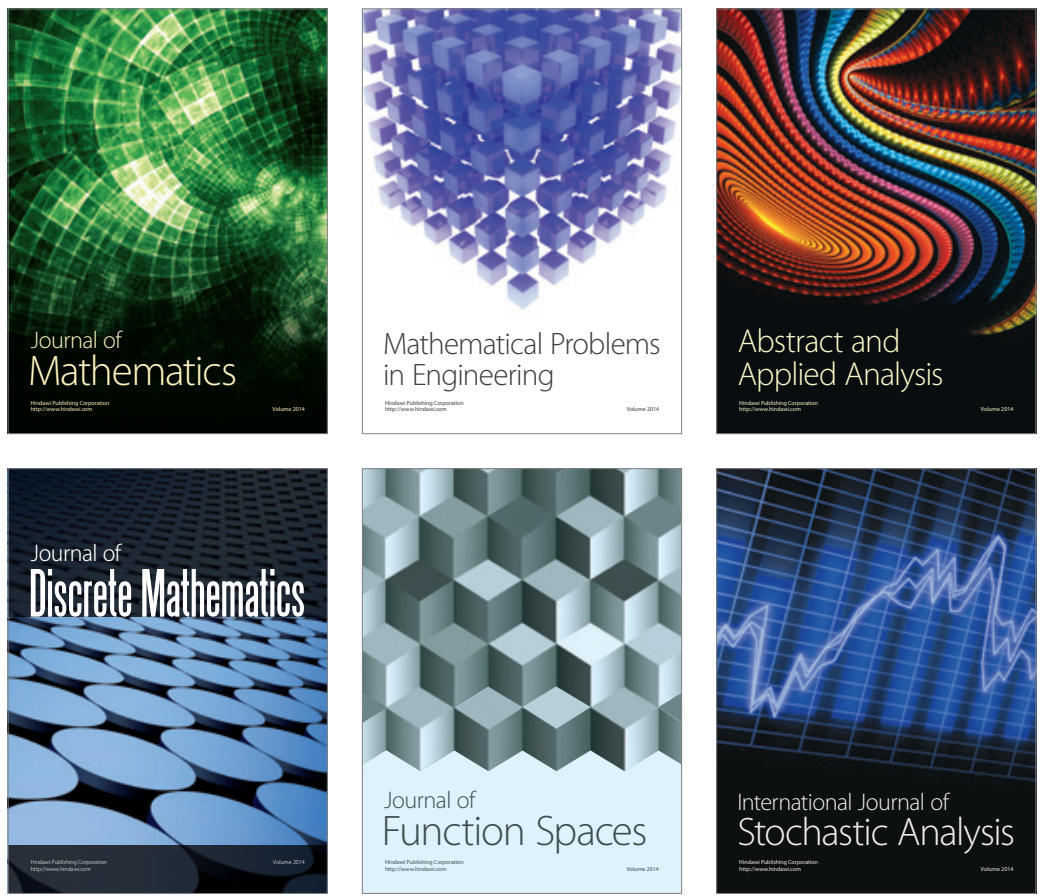

Journal of

Function Spaces

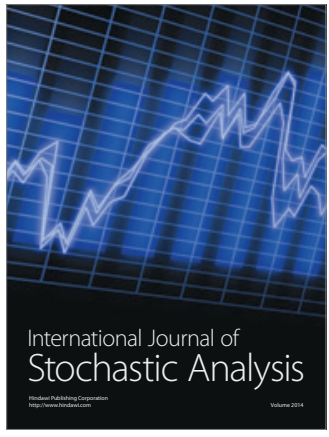

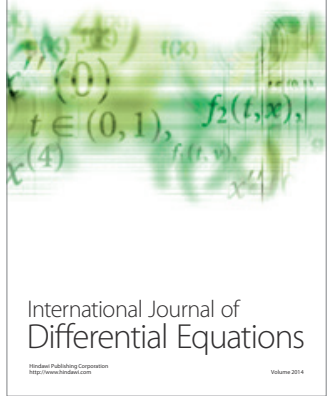
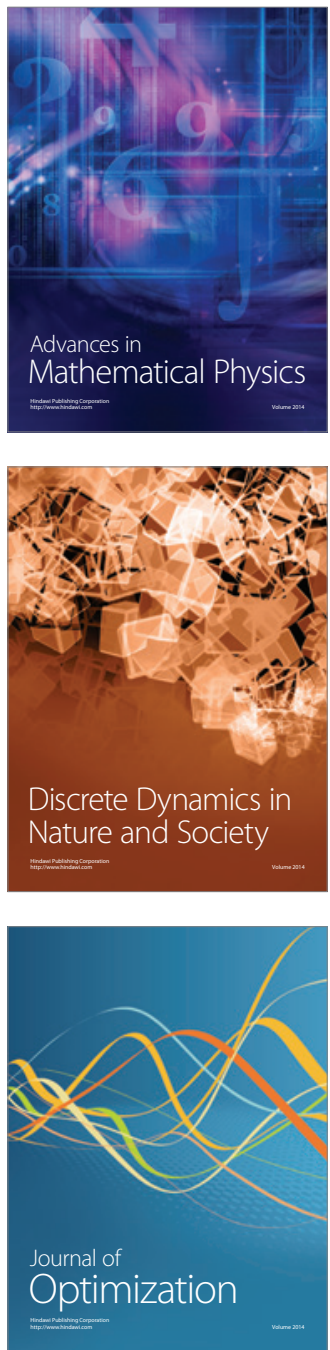